\title{
Microbiology Susceptibility Normal or Reference Range Indicator
}

National Cancer Institute

\section{Source}

National Cancer Institute. Microbiology Susceptibility Normal or Reference Range

Indicator. NCI Thesaurus. Code C162350.

A indication that microbiology susceptibility data is outside the normal or reference range. 